\title{
Comparação Entre as Modalidades de Caminhada e Corrida na Predição do Consumo Máximo de Oxigênio
}

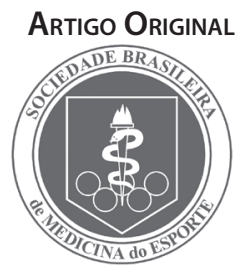

\section{Comparison Between the Gait and Running Modalities in the Oxygen Maximal Uptake Prediction}

Tony Meireles dos Santos $1,2,3$ Luis Felipe Larica Furtado 4 Leonardo Gonçalves Ribeiro 1,4 Leonardo Ferreira Cabral ${ }^{5}$ Jefferson da Silva Novaes ${ }^{4,6}$

1. Pro Health \& Performance - Rio de Janeiro (RJ)

2. Programa de Pós-Graduação em Educação Física da Universidade Gama Filho - Rio de Janeiro (RJ) 3. Curso de Educação Física das Faculdades Integradas Maria

Theresa - Niterói (RJ)

4. Programa de Pós-Graduação Stricto Sensu em Ciência da Motricidade Humana - PROCIMH - UCB (RJ)

5. Laboratório de Fisiologia do Exercício da Universidade Estácio de Sá - Campus Taquara - Rio de Janeiro (RJ)

6. Universidade Federal do Rio de Janeiro - UFRJ (RJ)

\section{Endereço para correspondência:}

Tony Meireles dos Santos

Pro Health \& Performance

Rua México, 168 - $12^{\circ}$ andar

sala 01 - Centro - RJ - CEP 20031-143

Tel. (21) 34194499

E-mail: tonyms@prohealth.com.br

Submetido em 25/07/2006

Versão final recebida em 30/07/2007

Aceito em 16/04/2008

\section{RESUMO}

Os testes ergométricos de exercício realizados em esteira podem ser desempenhados a partir das modalidades de caminhada e corrida, contudo, sem haver grandes elucidações ao comparar resultados de $\mathrm{VO}_{2 \text { máx }}$ obtidos nessas possibilidades de teste. Com o intuito de responder a essa questão, o presente estudo objetivou comparar a predição do $\mathrm{VO}_{2 \text { máx }}$ entre um protocolo escalonado máximo de caminhada e um de corrida, ambos elaborados de modo a proporcionar demandas metabólicas idênticas em cada estágio. Foram voluntários para o presente estudo 23 indivíduos (15 masculinos e oito femininos) com 27,7 \pm 7,6 anos de idade, $73,0 \pm 14,9 \mathrm{~kg}$ de massa corporal, 170,0 $\pm 1,0 \mathrm{~cm}$ de estatura, 19,3 $\pm 8,6 \%$ de gordura corporal, $24,7 \pm 3,3 \mathrm{~kg} \cdot \mathrm{m}^{-2}$ de índice de massa corporal e $44,1 \pm 6,0 \mathrm{~mL} \cdot \mathrm{kg}^{-1} \cdot \mathrm{min}^{-1}$ de potência aeróbia máxima. Um teste $t$ pareado não demonstrou diferenças significativas ( $p=0,364$; IC $95 \%=-2,2$ a 0,85) entre o protocolo de caminhada e o de corrida, apesar de a média dos valores do $\mathrm{VO}_{2 \text { máx }}$ na corrida ter sido $2,4 \%$ superior $\left(42,4 \mathrm{vs} .43,4 \mathrm{~mL} \cdot \mathrm{kg}^{-1} \cdot \mathrm{min}^{-1}\right)$. Com base nos resultados encontrados, conclui-se que a administração de diferentes estratégias de protocolos em esteira (caminhada ou corrida) não influenciou significativamente a estimativa do $\mathrm{VO}_{2 \text { max }}$ não interferindo na posterior tomada de decisão para a prescrição do treinamento cardiorrespiratório.

Palavras-chave: teste de exercício, teste em esteira, correndo, caminhando.

\section{ABSTRACT}

Exercise tests carried out on treadmills may be performed by running or gait; however, there is no agreement concerning the comparison of the $\mathrm{VO}_{2 \max }$ results obtained in these tests. Thus, the aim of this study was to compare the $\mathrm{VO}_{2 \max }$ prediction between both a running and a gait maximum scaled protocol, elaborated to cause identical metabolic demands in each stage. Twenty three subjects volunteered for this study - (15 men and eight women) aged $27.7 \pm 7.6$ years, $73.0 \pm 14.9 \mathrm{~kg}$ of body mass, $170.0 \pm 1.0 \mathrm{~cm}$ of height, $19.3 \pm 8.6 \%$ of body fat, $24.7 \pm 3.3{\mathrm{~kg} \times \mathrm{m}^{-2} \mathrm{of}}^{-1}$

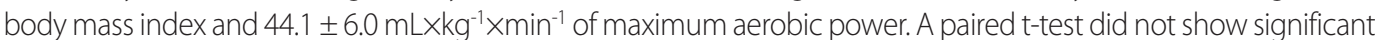
differences ( $p=0.364 ;$ RI $95 \%=-2.2$ to 0.85 ) between the gait and the running protocols; however, the mean value

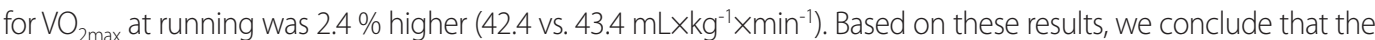
administration of different protocols on treadmill (gait or running) did not significantly influence the $\mathrm{VO}_{2 \text { max }}$ estimation, and hence did not interfere in the subsequent decision for the prescription of the cardiorespiratory training.

Keywords: exercise test, treadmill test, running, gait.

\section{INTRODUÇÃO}

A avaliação da aptidão física e das condições gerais de saúde tem sido recomendada como base para elaboração de programas de exercício de forma individualizada, segura e eficaz. A definição de quais parâmetros avaliar está vinculada ao próprio conceito de aptidão física em saúde, constituída pela composição corporal, força e resistência, flexibilidade e potência aeróbia(1).

Dentre esses parâmetros, a potência aeróbia máxima $\left(\mathrm{VO}_{2 \text { máx }}\right)$, definida como a maior taxa de oxigênio captado, transportado e utilizado pelos músculos recrutados, em associação com outros parâmetros fisiológicos e neuromusculares ${ }^{(2,3)}$, fornece importante informação sobre a capacidade de realização de atividades físicas de longa duração. Esse parâmetro é ainda fundamental para a tomada de decisão na prescrição de exercícios.

Apesar de alguns equipamentos para análise de variáveis de troca gasosa respiratória possibilitarem resultados considerados como padrão ouro para determinação do $\mathrm{VO}_{2 \text { máx }}$ sua aplicabilidade clínica e em centros de atividade física é inimaginável por questões geralmente associadas ao orçamento. Considerando a precisão de um dos equipamentos mais utilizados no Brasil(4-8), bem como a relação custo-benefício de utilização das equações metabólicas sugeridas pelo American College of Sports Medicine (ACSM)(1) , justifica-se a utilização das equações preditivas como estratégia de estimativa do consumo de oxigênio $\left(\mathrm{VO}_{2}\right)$ em atividades físicas diversas, incluindo a estimativa do $\mathrm{VO}_{2 \text { máx }}$.

Considerando isso, a predição do $\mathrm{VO}_{2 \text { máx }}$ a partir de equações indiretas tem sido uma prática empregada há tempos no estudo da saúde, aptidão física e performance atlética. Apesar do maior erro associado a essa estratégia, a relação custo benefício parece justificar sua aplicabilidade ${ }^{(9)}$. O ACSM ${ }^{(1)}$ indicou um erro aproximado de $7 \%$ na estimativa do consumo de oxigênio a partir de suas equações. Cabe considerar que as mesmas não foram desenvolvidas para a população nacional, nem tampouco existem estudos realizados nessa linha de investigação. 
Fatores como o ergômetro utilizado, nível de treinamento, adaptabilidade do testado, especificidade do movimento, tipo de protocolo, entre outros parâmetros, influenciam a relação entre $\mathrm{VO}_{2}$ estimado e o medido(10). Foram propostos nas últimas décadas diversos protocolos para diferentes tipos de ergômetros com o intuito de estimar o $\mathrm{VO}_{2 \text { máx }}$ A escolha por uma das opções disponíveis tem-se baseado em tradição, conveniência, familiaridade ou alguma combinação desses três determinantes ${ }^{(11)}$.

Das modalidades de teste ergométrico adotadas para a esteira, pode-se destacar na literatura o desenvolvimento de protocolos de corrida e caminhada ${ }^{(10)}$, sem, contudo, haver grandes elucidações ao comparar os resultados obtidos nessas duas modalidades de teste na predição do $\mathrm{VO}_{2 \text { máx }}$. Tradicionalmente, recomenda-se a modalidade de caminhada para indivíduos com menor competência cardiorrespiratória ou com indicação de monitoração eletrocardiográfica e de pressão arterial, enquanto a corrida, para os indivíduos melhor condicionados. Além disso, questões associadas à modalidade de atividade física praticada pelo testado também podem influenciar a conduta a ser adotada.

Considerando o exposto acima, o presente estudo objetivou comparar os valores de $\mathrm{VO}_{2 \text { máx }}$ preditos no teste de caminhada com aqueles obtidos em um teste de corrida, em um grupo de indivíduos fiscamente ativos.

\section{MÉTODOS}

\section{Sujeitos}

Foram voluntários para o presente estudo 23 indivíduos, sendo 15 do sexo masculino e oito do feminino, com idade entre 20 e 48 anos. Participaram do estudo funcionários e prestadores de serviços de uma universidade privada do Rio de Janeiro. Todos os sujeitos realizavam pelo menos 90 min semanais de atividades aeróbias. Como critério de inclusão, foram selecionados indivíduos classificados como de Risco Baixo segundo questionário de estratificação de risco desenvolvido pela American Heart Association e o ACSM ${ }^{(12)}$. Seriam eliminados do estudo indivíduos que apresentassem algum comprometimento osteomioarticular, apesar de tal condição não ter-se manifestado.

Todos os voluntários consentiram sua participação no presente estudo através da assinatura de um termo de consentimento livre e esclarecido elaborado em conformidade com a Resolução CNS 196/96 do Ministério da Saúde. Os procedimentos do presente estudo foram aprovados pela junta de ética em pesquisa do Laboratório de Fisiologia do Exercício da Universidade Estácio de Sá Campus Akxe ???????.

\section{Procedimentos experimentais}

A coleta dos dados foi realizada em duas sessões distintas. Na primeira visita ao laboratório, os voluntários preencheram os formulários de consentimento e questionário de estratificação de risco, foram submetidos a uma bateria de medidas antropométricas, contendo massa (balança digital Filizola, Filizola, RJ, Brasil), estatura e dobras cutâneas (Slim Guide, Rosscraft, BC, Canada).

Para a realização das medidas, foram adotadas as normas sugeridas pela International Society for Advancement of Kinanthropometry ${ }^{(13,14)}$, exceto a dobra supra-ilíaca para mulheres, que seguiu as normas de Jackson et al.(15), com posterior determinação da composição corporal $^{(15-17)}$ (percentual de gordura, massa de gordura e massa livre de gordura) e índice de massa corporal.

Após o término das medidas antropométricas, os voluntários foram submetidos a um teste para predição do $\mathrm{VO}_{2 \text { Máx }}$ a partir de um dos protocolos de teste em esteira (esteira ATL, Imbrasport, RS, Brasil) de caminhada ou corrida determinado aleatoriamente. Um sorteio possibilitou que 11 voluntários começassem pelo protocolo de caminhada e 12 pelo de corrida. Na segunda sessão, os avaliados realizaram o outro teste aeróbio, a partir do protocolo ainda não utilizado.
Seguindo a recomendação do estudo realizado por Giancristoforo et al. ${ }^{(18)}$, foram elaborados dois protocolos (caminhada e corrida) adequados às características de condicionamento físico do grupo investigado no presente estudo e com o objetivo de proporcionar a mesma demanda metabólica (em METs) por estágio, calculada a partir das equações sugeridas pelo ACSM(1).

Os protocolos utilizados nos testes ergométricos de corrida e caminhada foram do tipo escalonado máximo, com incrementos regulares a cada dois minutos de aproximadamente $2,2 \mathrm{~mL} \cdot \mathrm{kg}^{-1} \cdot \mathrm{min}^{-1}$, iniciandose a $29,3 \mathrm{~mL} \cdot \mathrm{kg}^{-1} \cdot \mathrm{min}^{-1}$. No teste de caminhada, após aquecimento a $1,25 \mathrm{~m} \cdot \mathrm{s}^{-1}\left(4,5 \mathrm{~km} \cdot \mathrm{h}^{-1}\right)$ e $0 \%$ de inclinação com três minutos de duração, manteve-se a velocidade constante em $1,53 \mathrm{~m} \cdot \mathrm{s}^{-1}\left(5,5 \mathrm{~km} \cdot \mathrm{h}^{-1}\right)$ com incrementos de 1,3\% a cada dois minutos a partir da inclinação de $10 \%$. No teste de corrida, após o mesmo aquecimento já descrito para a caminhada, a inclinação foi mantida em $2 \%$, com a velocidade aumentada a cada dois minutos em $0,17 \mathrm{~m} \cdot \mathrm{s}^{-1}\left(0,6 \mathrm{~km} \cdot \mathrm{h}^{-1}\right)$ a partir da velocidade inicial de $1,97 \mathrm{~m} \cdot \mathrm{s}^{-1}\left(7, \mathrm{~km} \cdot \mathrm{h}^{-1}\right)$.

Foram adotados os critérios de interrupção de teste de esforço sem fins diagnósticos para indivíduos aparentemente saudáveis sugeridos pelo ACSM $^{(1)}$. Nos momentos finais de cada teste, os voluntários receberam estímulos verbais dos avaliadores para permanecer em atividade até o limite máximo percebido.

Para a determinação do $\mathrm{VO}_{2 \text { máx }}$ em ambos os protocolos, utilizouse o último estágio completado pelo testado. Caso algum voluntário interrompesse o teste antes de sua finalização, era considerado o estágio anterior para o cálculo e registro do $\mathrm{VO}_{2 \text { máx }}$ A partir da velocidade, inclinação e modalidade do teste, os valores de $\mathrm{VO}_{2 \text { máx }}$ foram preditos com base nas equações do ACSM $^{(1)}$.

Ao final de cada estágio, foram registradas a freqüência cardíaca (FC) (Vantage, Polar Electro Oy, Kempele, Finlândia) e a escala subjetiva de esforço(19). Após o término do teste, os voluntários realizaram uma recuperação ativa por um período mínimo de quatro minutos com a mesma velocidade e inclinação adotados no momento de aquecimento do teste.

\section{Análise estatística}

Foi utilizada estatística descritiva (média \pm desvio-padrão) para caracterização da amostra. A verificação de diferenças para a variável dependente $\left(\mathrm{VO}_{2 \text { máx }}\right)$ entre os testes de corrida e caminhada, para homens, mulheres e o total da amostra, foi estabelecida a partir do teste $t$ pareado, tendo sido adotado o nível de significância de $\alpha \leq$ 0,05. Todas as análise foram realizadas no SPSS v. 12.0 (SPSS Inc., Chicago, IL, EUA).

\section{RESULTADOS}

A tabela 1 contém as principais características do grupo investigado. Comparada com as normas sugeridas pelo $\operatorname{ACSM}^{(1)}$, a média do percentual de gordura para os homens apresentou-se abaixo do percentil 60 , sendo classificado como Na Média e, para as mulheres, abaixo do percentil 20, sendo classificado como Bem Abaixo da Média. Já a média do $\mathrm{VO}_{2 \text { máx }}$ da amostra masculina apresentou-se abaixo do percentil 70, sendo classificado como Na Média e o da amostra feminina, acima do percentil 70, sendo classificado como Acima da Média.

Todos os testes de esforço foram interrompidos por determinação dos testados em virtude de terem alcançado a exaustão. $O V_{2} O_{2 m a ́ x}$ estimado a partir dos diferentes protocolos utilizados encontra-se na figura 1 . $O$ resultado do teste $t$ não demonstrou diferença significativa ( $p=0,364$; IC $95 \%=-2,2$ a 0,85) entre o protocolo de caminhada e o de corrida, apesar de a média dos valores do $\mathrm{VO}_{2 \text { máx }}$ na corrida ter sido $2,4 \%$ superior $\left(42,4 \pm 6,2\right.$ vs. $\left.43,4 \pm 5,5 \mathrm{~mL} \cdot \mathrm{kg}^{-1} \cdot \mathrm{min}^{-1}\right)$. 
Tabela 1. Características da amostra investigada (média \pm desvio-padrão)

\begin{tabular}{|c|c|c|c|}
\hline Variáveis & $\begin{array}{l}\text { Homens } \\
(n=15)\end{array}$ & $\begin{array}{l}\text { Mulheres } \\
(n=8)\end{array}$ & $\begin{array}{c}\text { Amostra total } \\
\quad(n=23)\end{array}$ \\
\hline Idade (anos) & $27,8 \pm 7,7$ & $27,5 \pm 8,1$ & $27,7 \pm 7,6$ \\
\hline Massa (kg) & $77,9 \pm 12,9$ & $63,7 \pm 14,5$ & $73,0 \pm 14,9$ \\
\hline Estatura (cm) & $175,6 \pm 6,5$ & $163,3 \pm 6,9$ & $170,0 \pm 1,0$ \\
\hline IMC $\left(\mathrm{kg} \cdot \mathrm{m}^{-2}\right)$ & $25,1 \pm 2,9$ & $23,8 \pm 4,1$ & $24,7 \pm 3,3$ \\
\hline Gordura percentual $(\%)^{*}$ & $15,7 \pm 5,9$ & $25,9 \pm 9,2$ & $19,3 \pm 8,6$ \\
\hline Massa Livre de Gordura (kg) & $65,3 \pm 9,4$ & $46,3 \pm 6,7$ & $58,7 \pm 12,5$ \\
\hline $\mathrm{VO}_{2 \text { máx }}\left(\mathrm{mL} \cdot \mathrm{kg}^{-1} \cdot \mathrm{min}^{-1}\right)^{\dagger}$ & $47,1 \pm 4,7$ & $38,6 \pm 4,1$ & $44,1 \pm 6,0$ \\
\hline
\end{tabular}

* Segundo a equação de Siri(17) com base na densidade corporal de Jackson et al. ${ }^{(15)}$ e Jackson e Pollo$\mathrm{Ck}^{(16) ;}{ }^{\dagger}$ valores médios estabelecidos a partir dos resultados individuais nos dois testes realizados.

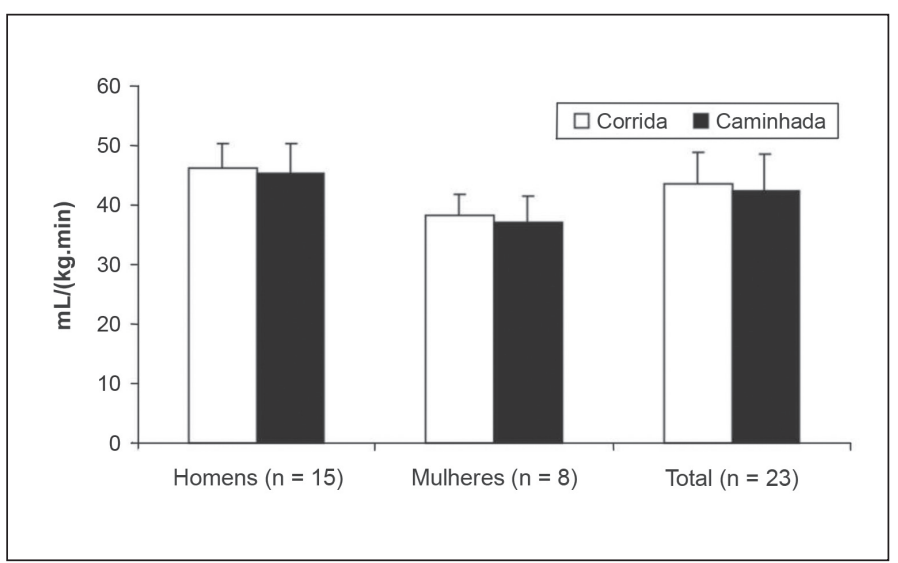

Figura 1. Comparação dos resultados de $\mathrm{VO}_{2 \text { máx }}$ estimados nos protocolos escalonados de caminhada e corrida.

\section{DISCUSSÃO}

O principal achado do presente estudo foi a constatação de não existir diferença entre o $\mathrm{VO}_{2 \text { máx }}$ da caminhada e corrida quando os protocolos utilizados apresentam demanda metabólica idêntica por estágio.

Foster et al.(20) foram os primeiros a sugerir a estratégia do presente estudo como método de determinação do $\mathrm{VO}_{2 \text { máx }}$ de forma dissociada a protocolos tradicionais como o de Bruce ou Balke. Em consonância com o presente estudo, os autores sugeriram a idéia de individualização do protocolo de acordo com as características dos testados. Os índices de determinação $(R)$ para a realização do teste com e sem o apoio das mãos foram de 0,995 (EPE de 4,8 $\mathrm{mL} \cdot \mathrm{kg}^{-1} \cdot \mathrm{min}^{-1}$ ) e 0,833 (EPE de $\left.4,4 \mathrm{~mL} \cdot \mathrm{kg}^{-1} \cdot \mathrm{min}^{-1}\right)$, respectivamente. Apesar da não comparação entre as modalidades de caminhada e corrida, os autores demonstraram a possibilidade e necessidade de flexibilização dos protocolos de acordo com as características dos testados.

Por princípio, um protocolo para estimativa do $\mathrm{VO}_{2 \text { máx }}$ deve levar em consideração o nível de condicionamento físico do indivíduo a ser testado, adequando assim à taxa de trabalho inicial e a sua razão de incremento ${ }^{(11,18)}$. A individualização de protocolos de teste de exercício baseia-se em três princípios básicos da ergometria(10,11,18), a saber: a) duração total do teste de aproximadamente 10 min (8 a 12 min); b) razão padronizada de sobrecarga por estágio; e c) início do protocolo com cargas equivalentes a aproximadamente 40-60\% da carga máxima tolerada por indivíduos com nível de condição física moderado. O não respeito a tais preceitos pode acarretar superestimativa do $\mathrm{VO}_{2 \text { máx }}$ em virtude de incremento elevado ou do curto tempo de cada estágio(21).
Vale ressaltar que a individualização dos protocolos possibilita maior precisão nas estimativas do $\mathrm{VO}_{2 \text { máx }}$ (especialmente em indivíduos cardiopatas), maior sensibilidade para condições de reteste, identificação de doença arterial coronariana ${ }^{(11)}$ e determinação dos limiares metabólicos $^{(22)}$. Entretanto, Starling et al. ${ }^{(23)}$ demonstraram achados diferente. Utilizando o protocolo de Bruce, que está associado a incrementos abruptos a cada estágio de teste, os autores observaram maior sensibilidade na detecção de respostas eletrocardiográficas isquêmicas do que nos protocolos associados a incrementos mais brandos como, por exemplo, o de Naughton (caminhada).

A utilização das equações metabólicas na estimativa do $\mathrm{VO}_{2} \mathrm{em}$ testes aeróbios tem sido questionada nos últimos tempos. Investigando a utilização dessas equações em testes progressivos máximos de corrida, Ruiz e Sherman ${ }^{(24)}$ observaram resultados diferentes. Comparando os valores estimados com a medida direta, os autores observaram superestimativa na ordem de $4,7 \mathrm{~mL} \cdot \mathrm{kg}^{-1} \cdot \mathrm{min}^{-1}$. Apesar da correlação significativa entre os valores estimados e medidos de $\mathrm{VO}_{2 \text { máx }}(r=0,771)$, os resultados preditos apresentaram-se acima do valor real em aproximadamente $88 \%$ dos testes realizados.

Hall et al. ${ }^{(25)}$ investigaram a aplicação das equações metabólicas do ACSM, incluindo as utilizadas no presente estudo, na estimativa do dispêndio energético de atividade com velocidades padronizadas de corrida e caminhada com duração equivalente à da cobertura de distância de $1.600 \mathrm{~m}$. Os resultados indicaram adequada predição do dispêndio energético total para ambas as equações, com um erro total (análise de Bland-Altman) na caminhada e na corrida de aproximadamente $4,1 \%$.

A provável discrepância entre o trabalho de Ruiz e Sherman ${ }^{(24)}$ e Hall et al. ${ }^{(25)}$ está no uso inadequado das equações do ACSM quando aplicadas em testes progressivos máximos a partir de cargas demasiadamente curtas (um a dois minutos) em cada estágio de teste. Tais questões foram adequadamente discutidas por Swain et al.(26), demonstrando maior precisão das equações quando utilizadas em condição de estado estável (seis minutos de duração por estágio).

Com base nos argumentos apresentados ${ }^{(24-26)}$, pode-se concluir que o principal problema associado à utilização das equações preditivas está relacionado ao seu uso inadequado, em especial quando aplicadas a cargas com duração curta (um a três minutos). O presente estudo utilizou cargas por estágio com duração de dois minutos, causando tendência à superestimativa do $\mathrm{VO}_{2 \text { máx }}$ como já destacado anteriormente ${ }^{(24)}$. Entretanto, como tal condição foi adotada tanto para o protocolo de corrida quanto para o de caminhada, o erro sistemático promovido por essa estratégia não influenciou os resultados encontrados no presente estudo.

Alguns trabalhos tradicionais investigaram as diferenças existentes entre o consumo máximo de oxigênio em testes de corrida vs. caminhada. Froelicher et al.(27), utilizando 15 voluntários que foram submetidos aleatoriamente aos protocolos de Bruce, Balke e Taylor, não observaram diferenças significativas entre os protocolos de Balke e Bruce $\left(42,8 \mathrm{~mL} \cdot \mathrm{kg}^{-1} \cdot \mathrm{min}^{-1}\right.$ vs. $\left.44,3 \mathrm{~mL} \cdot \mathrm{kg}^{-1} \cdot \mathrm{min}^{-1}\right)$, apesar da ligeira superioridade da média do $\mathrm{VO}_{2 \text { máx }}$ no protocolo de Bruce $(3,4 \%)$.

Pollock et al.(28) encontraram em 51 indivíduos (29 destreinados e 22 treinados), submetidos a um protocolo de caminhada (Balke) e três de corrida, diferença média do $\mathrm{VO}_{2 \text { máx }}$ de 3,5\% (1,5\% para Bruce, 3,2\% para Ellestad e 5,9\% para Astrand), sendo observada diferença estatisticamente significativa somente para o protocolo de Astrand $(p<0,05)$.

Outro estudo realizou comparação do $\mathrm{VO}_{2 \text { pico }}$ utilizando dois protocolos, um de caminhada com inclinação progressiva e outro de corrida com velocidade progressiva, entre dois grupos de atletas, sendo um de corredores e o outro de jogadores de squash ${ }^{(29)}$. Os jogadores de squash atingiram valores $4,6 \%$ mais altos no teste de corrida do que no 
teste de caminhada, enquanto os corredores obtiveram apenas 1,95\% de diferença entre as duas modalidades. Quando calculada a média dos resultados obtidos do $\mathrm{VO}_{2 \text { pico }}$ entre os dois grupos, foram encontrados valores 3,3\% mais altos no protocolo de corrida em comparação com o protocolo de caminhada.

Em consonância com evidências apresentadas ${ }^{(27-29)}$, os dados do presente estudo demonstraram diferença não significativa de 2,4\% entre as modalidades de caminhada e corrida. Para indivíduos adultos, a maior parte das evidências, incluindo o presente estudo, sugere discreta diferença, na grande maioria não significativa, entre as abordagens de caminhada e corrida.

Somente no trabalho de Marinov et al. ${ }^{(30)}$, utilizando crianças como voluntárias, observou-se diferença significativa entre o protocolo de Balke tradicional (caminhada progressiva contínua) e o de Bruce (caminhada com posterior corrida progressiva contínua), da ordem de 34,8\%. A discrepância observada foi reduzida a partir da modificação do protocolo de Balke, a fim de promover a interrupção do teste em tempo mais curto, resultando em diferença de 20,9\%. Tal redução nas diferenças pode ter ocorrido pela adequação do protocolo às características do grupo testado, indicando a importância da individualização de protocolos de teste de exercício.

A menor diferença entre as duas modalidades de protocolos observada no presente estudo pode ter sido causada pela padronização dos incrementos de carga utilizados. Para indivíduos adultos, as diferenças entre as modalidades de caminhada e corrida parecem não representar dado de relevância para interpretação dos resultados do teste, bem como prescrição de exercícios.

Com base nos resultados encontrados, conclui-se que a adoção do protocolo de corrida ou caminhada não proporcionou diferença na predição do $\mathrm{VO}_{2 \text { máx. }}$ Os resultados do presente estudo parecem indicar que, apesar de não serem significativas, as pequenas diferenças observadas entre os dois protocolos podem ser reduzidas utilizandose um fator de correção de 2,4\%, caso a orientação da atividade física utilize modalidade diferente daquela testada.

Uma limitação do presente estudo, no que se refere à determinação do $\mathrm{VO}_{2 \text { máx }}$ foi a adoção de métodos indiretos e não a medida direta do consumo de oxigênio. Apesar de em nada influenciar na tomada de decisão para a prescrição de exercícios, uma vez que tais estratégias também se baseiam em métodos indiretos, para fins de maior precisão nas conclusões alcançadas, recomenda-se a replicação do presente estudo utilizando a medida direta do consumo de oxigênio. Tal recomendação sustenta-se também na possível superestimativa do $\mathrm{VO}_{2 \text { máx }}$ em ambos os protocolos investigados, como sugerido por Ruiz e Sherman ${ }^{(24)}$.

Sugere-se ainda que, em futuros estudos, sejam investigadas diferenças entre protocolos com cargas de incrementos não padronizadas, bem como a partir de amostras diferenciadas das estudadas neste experimento, como em crianças, idosos e atletas.

\section{AGRADECIMENTOS}

Os autores agradecem aos voluntários que gentilmente participaram deste estudo. Agradecimentos ainda à amiga Marta Inez Rodrigues Pereira e ao mestre Edmundo Vieites Novaes, pelas importantes contribuições na revisão deste manuscrito, e a Leonardo Alevatto, pela versão do Abstract.

Todos os autores declararam não haver qualquer potencial conflito de interesses referente a este artigo.

\section{REFERÊNCIAS BIBLIOGRÁFICAS}

1. American College of Sports Medicine. ACSM's Guidelines for Exercise Testing and Prescription. 7th ed. Baltimore: Lippincott Williams and Wilkins, 2005.

2. Noakes TD. Physiological models to understand exercise fatigue and the adaptations that predict or enhance athletic performance. Scand J Med Sci Sports 2000;10:123-45.

3. Paavolainen L, Hakkinen K, Hamalainen I, Nummela A, Rusko H. Explosive-strength training im proves $5-\mathrm{km}$ running time by improving running economy and muscle power. J Appl Physio 1999:86:1527-33.

4. Segal KR, Chatr-Aryamontri B, Guvakov D. Validation of a new portable metabolic measurement system. Med Sci Sports Exerc 1994;26:S54.

5. Clure C, Watts PB, Gallagher P, Hill R, Humphreys S, Wilkins B. Accuracy of the TEEM 100 metabolic analyzer during maximum oxygen uptake testing of Nordic skiers. Med Sci Sports Exerc 1995;27:S86

6. Melanson EL, Freedson PS, Hendelman D, Debold E. Reliability and validity of a portable metabolic measurement system. Can J Appl Physiol 1996;21:109-19.

7. Wideman L, Stoudemire NM, Pass KA, McGinnes CL, Gaesser GA, Weltman A. Assessment of the aerosport TEEM 100 portable metabolic measurement system. Med Sci Sports Exerc 1996;28:509-15.

8. Novitsky S, Segal KR, Chatr-Aryamontri B, Guvakov D, Katch VL. Validity of a new portable indirect calorimeter: the AeroSport TEEM 100. Eur J Appl Physiol Occup Physiol 1995;70:462-7.

9. Swain DP. Energy cost calculations for exercise prescription: an update. Sports Med 2000;30:17-22.

10. Fletcher GF, Balady GJ, Amsterdam EA, Chaitman B, Eckel R, Fleg J, et al. Exercise standards for testing and training: a statement for healthcare professionals from the American Heart Association. Circulation 2001;104:1694-740.

11. Myers J, Bellin D. Ramp exercise protocols for clinical and cardiopulmonary exercise testing. Sports Med 2000;30:23-9

12. American College of Sports Medicine Position Stand and American Heart Association. Recommendations for Cardiovascular Screening, Staffing, and Emergency Policies at Health/Fitness Facilities. Med Sci Sports Exerc 1998:30:1009-18

13. Norton K, Olds T. Anthropometrica. Sidney: University of New South Wales Press, 1996

14. Ross WD, Carr RV, Carter JEL. Anthropometry Illustrated. 1st ed. Toronto, Canada: Turnpike Electronic Publications Inc., 1999

15. Jackson AS, Pollock ML, Ward A. Generalized equations for predicting body density of women. Med Sci Sports Exerc 1980;12:175-81.
16. Jackson AS, Pollock ML. Generalized equations for predicting body density of men. Br J Nutr 1978;40:497-504

17. Siri WE. Body Composition from fluid spaces and density. In: Brozek J, Henschel A, editors. Techniques of measuring body composition. Washington DC: National Academy of Science, 1961;233-44.

18. Giancristoforo LN, Miyamoto GA, Santos TM. Proposta de adequação do testado a diferentes protocolos escalonados de teste de esforço cardiorrespiratório. $23^{\circ}$ Simpósio Internacional de Ciência do Esporte. Tema livre XV - Fisiologia, Treinamento e Atividade Física 78. São Paulo: Celafiscs 2000.

19. Borg G. Borg's Perceived Exertion and Pain Scales. Champaing, IL: Human Kinetics, 1998.

20. Foster C, Crowe AJ, Daines E, Dumit M, Green MA, Lettau S, et al. Predicting functional capacity during treadmill testing independent of exercise protocol. Med Sci Sports Exerc 1996;28:752-6.

21. Montoye HJ, Ayen T, Nagle F, Howley ET. The oxygen requirement for horizontal and grade walking on a motor-driven treadmill. Med Sci Sports Exerc 1985;17:640-5.

22. Santos TM, Gomes PSC. Influência de dois protocolos e cinco critérios na relação entre os limiares ventilatórios e láticos. Artus - Rev Ed Fis Desp 1998:18:53-65.

23. Starling MR, Crawford MH, O'Rourke RA. Superiority of selected treadmill exercise protocols predischarge and six weeks postinfarction for detecting ischemic abnormalities. Am Heart J 1982;104:1054-60,

24. Ruiz A, Sherman NW. An evaluation of the accuracy of the American College of Sports Medicine metabolic equation for estimating the oxygen cost of running. J Strength Cond Res 1999:13:21923.

25. Hall C, Figueroa A, Fernhall B, Kanaley JA. Energy expenditure of walking and running: comparison with prediction equations. Med Sci Sports Exerc 2004;36:2128-34

26. Swain DP, Parrott JA, Bennett AR, Branch JD, Dowling EA. Validation of a new method for estimating VO2max based on VO2 reserve. Med Sci Sports Exerc 2004;36:1421-6.

27. Froelicher VF Jr, Brammell H, Davis G, Noguera I, Stewart A, Lancaster MC. A comparison of three maximal treadmill exercise protocols. J Appl Physiol 1974;36:720-5.

28. Pollock ML, Bohannon RL, Cooper KH, Ayres JJ, Ward A, White SR, et al. A comparative analysis of four protocols for maximal treadmill stress testing. Am Heart J 1976;92:39-46.

29. St Clair Gibson A, Lambert MI, Hawley JA, Broomhead SA, Noakes TD. Measurement of maximal oxygen uptake from two different laboratory protocols in runners and squash players. Med Sci Sports Exerc 1999;31:1226-9.

30. Marinov B, Kostianev S, Turnovska T. Modified treadmill protocol for evaluation of physical fitness in pediatric age group--comparison with Bruce and Balke protocols. Acta Physiol Pharmacol Bulg 2003:27:47-51. 